\title{
Physics on social media: impact and interaction to deal with fast communication
}

\author{
Francesca Mazzotta ${ }^{1}$ \\ INFN Communications Office \\ Piazza dei Caprettari 70, 00186 Roma (RM), Italy \\ E-mail: francesca.mazzotta@presid.infn.it
}

Traditional communication channels are now co-living with new ones, such as social media. These are channels through which communication is fast, impactful and interactive. It's commonly thought that they are mainly used by young people, but now there is an additional increasingly high demand of reliable and easy to understand information on social media by adults. Scientific research results need hence to be easy to find on these channels by those that are familiar with them but also by those publics that are starting now to approach these channels.

These are the reasons why, in order to provide accurate and reliable information, scientific research institutes need to be able to adopt a communication strategy appropriate for social media. These channels require indeed an approach that is different from the 'traditional communication' one, they need to provide information quickly, interactively and they can never be silent, this means that even when scientific institutes are not able to release breaking news they need to provide users of information that is not yet very well known. At INFN, these three requirements have led to the creation of new communication strategies that are based on two concepts: interaction and impact. INFN has created a series of weekly appointments that through an images-storytelling unveil the work that is done daily at the institute. Moreover, it has planned a series of interactive interviews, Facebook lives, that allow the public to directly ask questions to the first actors in the research field.

To reach an increasingly higher public and to meet the new public demands scientific research institutes have hence to be strongly present on these new communication channels, social media.

EPS-HEP2017, European Physical Society conference on High Energy Physics

5-12 July 2017

Venice, Italy

\footnotetext{
${ }^{1}$ Speaker

(C) Copyright owned by the author(s) under the terms of the Creative Commons

Attribution-NonCommercial-NoDerivatives 4.0 International License (CC BY-NC-ND 4.0).
} 


\section{Why can social media not be ignored?}

Social media are now part of our everyday life. An increasingly higher number of people is using them.[1] In addition to that, the public that is now using social media is very diversified.[2] Hence, to reach these new publics and meet their demands scientific research institutes need to be strongly present on these new communications channels and therefore they need to design new and appropriate communication strategies. The strategy adopted recently by INFN, the Italian Institute for Nuclear Physics, is presented in relation to the new needs that social media rise.

\subsection{Bridge users-news}

Social media can also be considered as a hook that leads people to news or other more in depth articles.[3] This is proven by a practical example. During the European Physical Society Conference on High Energy Physics that took place in Venice in July 2017, there was a discovery that was extensively highlighted on different kinds of media channels: the discovery of a particle never detected before, the Xicc++ particle. On this occasion an online article was published on asimmetrie.it, the website of the INFN Journal "Asimmetrie". On that day, 6 July 2017, the above mentioned website registered 1,128 entries and $74 \%$ of those came from Facebook (Figure 1).

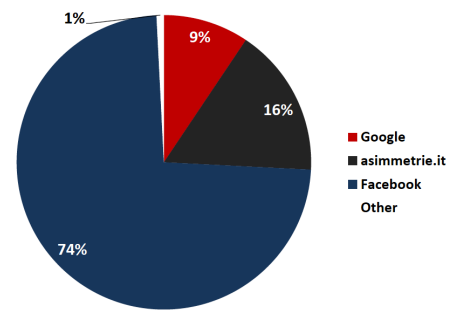

Figure 1. Entries on asimmetrie.it website on 6/07/2017

\subsection{Wider outreach than traditional online media}

The same article was used to compare the outreach of an article uploaded on the website (asimmetrie.it), and the outreach of an article when it was shared on social media. On Facebook the article reached 30,359 views, compared to the 1,128 pageviews of the website. Therefore in this occasion, the exact same news had a much wider outreach on social media than on traditional online media channels.

\subsection{Reactions and interactions in real time}

Social media can give to scientific insitutions the chance to collect reactions from the users in real or near-real time.[3] An example, in INFN experience, is given by Facebook lives. When you record a live video on Facebook you receive reactions in real time, and thanks to them you can tune your answers, and you can receive comments or questions in real time that can help making the video easy to understand by the public. This is particularly useful when talking about topics the public may be not familiar with, such as particle physics ones. 


\section{Chosen channels}

In Italy, the most widely used social media channels are Facebook, Youtube, Instagram and Twitter.[4] This is the reason why INFN is mainly designing communications strategies appropriate fore three of these channels. The communication strategies adopted for three of these channels (Facebook, Instagram and Twitter) are presented.

\subsection{Facebook}

INFN is on Facebook since February 2009. Between September 2016 and 2017, INFN Facebook page started to grow more steadily, it went from 14,061 to 18,534 likes on the page. This growth is mainly due to the adoption of a more intense social media strategy starting from January 2017. However, also in the previous year INFN Facebook page experienced an extensive but more sudden growth: for example, on the day of the announcement of the first detection of gravitational wave the page gained 1,244 likes. It has also to be noted that the outreach of the page is much wider than its followers, for example in 2017 on a single day (13 May 2017) the page reached 383,869 people.

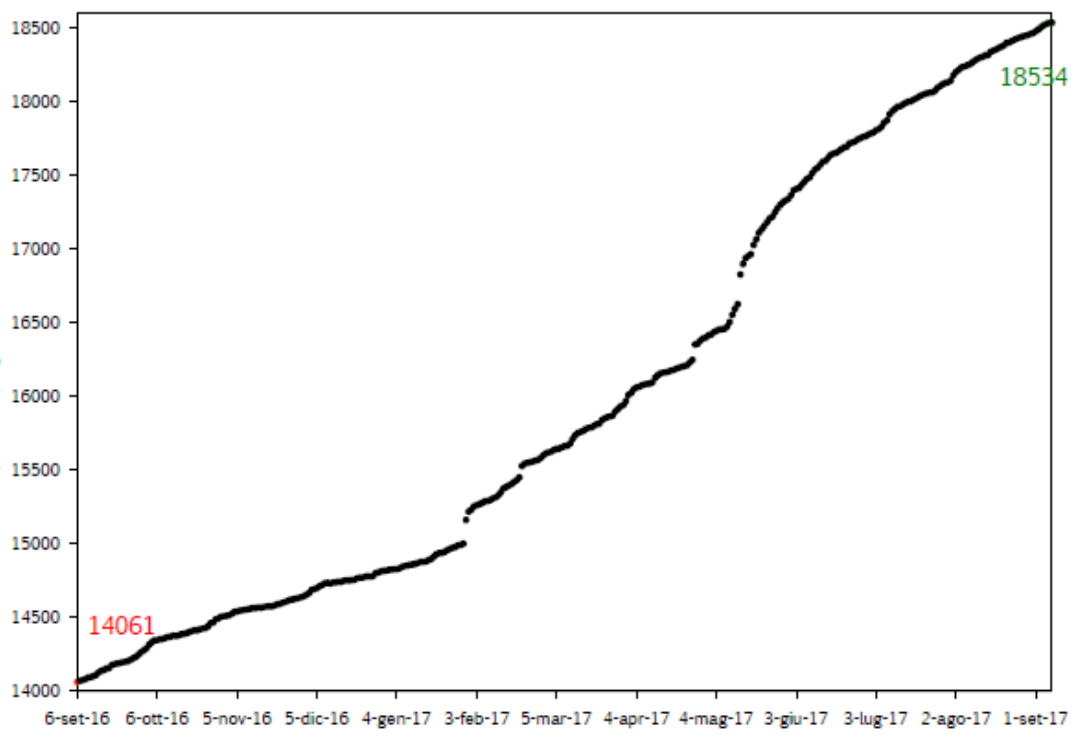

Figure 2. Number of likes on INFN Facebook page from 6/09/2016 to 6/09/2017

\subsection{Instagram}

INFN joined Instagram at the end of January 2017, and in only eight months its account, "infn_insigths", gained 1,180 followers. Instagram has been growing a lot in Italy during the past year. Today 14 milion Italian users use this channel at least once a month, a much higher number than last year's one (9 milion Italian users).[5]

\subsection{Twitter}

INFN joined Instagram in November 2009 and it now has 5,375 followers. The outreach of its tweet is however wider than the number of followers; in 2017 a single INFN tweet reached as far as 67,400 users. 


\section{Activities and discussion}

In order to respond to the new demands of social media, such as the need to provide information quickly, interactively and regularly, INFN has adopted of series of communication strategies and initiatives, on top regularly posting news about the insitute's research results.

\subsection{Schedule}

Social media can never be silent, this means that even when there are no breaking news, different kinds of information can be provided to the users. This can also be a chance to present minor or less known projects a scientific institute is working on. INFN is adopting this approach on the scheduling of the posts of its Instagram profile. Everyweek three posts are published within three different sections: experiments, laboratories and traces. In this way, the account is always active and all the different INFN activities and divisions can be presented.

\subsection{Lives}

To facilitate interactions of the users with the posts, a new product thought exclusively for social media was launched: Facebook lives. During Facebook lives two experts are interviewed directly by the users. The response of the users to the lives has been great, the first INFN one was launched in February 2017 during the dedication ceremony of the upgraded version of the gravitational waves' detector, Advance VIRGO. It had approximately 6,000 views, and after that six more Facebook lives were recorded reaching up to 14,000 views. On top of the views, users were keen on interacting with the experts before and during the live event. INFN last Facebook live, recorded on October 2017 right after the announcement of the winners of the 2017 Nobel Prize for physics, received over 600 reactions.

\subsection{Campaigns and collaborations}

In 2017 INFN joined existing social media campaigns, designed new ones on its own and in collaboration with other scientific institutions.

From April to June 2017, on the occasion of LHC 2017 restart, INFN joined CERN social media campaign \#WhatsUpLHC and launched a new campaign \#LHCItalia, which aimed at highlighting the contributions of Italian and INFN scientists to LHC. As part of these campaigns a series of video-interviews was posted on Facebook and Twitter, reaching up to 68'500 people.

Furthermore in 2017 INFN, CERN and Fermilab jointly designed a social media campaign \#IcarusTrip. In June 2017 the largest liquid-argon neutrino dector in the world that began its life at INFN (at Gran Sasso National Labotories) moved from CERN to Fermilab where it arrived at the end of July 2017. The social media campaign was aiming at following the detector's journey and it resulted to be successful, three Facebook lives were recorded during the campaign and they reached up to 64,000 views.

\subsection{Opportunities}

Since communication on social media is fast and impactul, opportunities need to be recognised and taken to widen the public to talk to. In February 2016, on the occasion of the announcement of the fist discovery of gravitational waves Matteo Renzi, the former Italian prime minister, published a tweet to congratulate INFN research that INFN promptly retweeted. While in May 2017, INFN hosted an event with a group of popular Italian YouTubers (The 
Jackal), on that occasion a short video about dark matter was shot and promptly published on Facebook. On that day, 13 May 2017, INFN Facebook page reached 383'869 users.

\section{Conclusions}

Social media are rising a series of diverse communicatios needs such as the need of a more quick, interactive and regular communication. INFN is hence strengthening its presence on social media by having a tight weekly schedule, designing new communication products such as Facebook lives and working on joint social media campaigns. However since social media are always changing communciations channels, in the future these communication strategies may be changed or implemented according to the upcoming needs.

\section{References}

[1] G. Riva, I social network, Il Mulino Bologna 2016: 194

[2] H. Gil de Zúñiga, N. Jung, S. Valenzuela, Social media use for news and individuals' social capital, civic engagement and political participation, JCMC. 17(3), 319-336

[3] L. Safko, D. K. Brake, The social media bible, ExecuGo media 2009.

[4] https://www.wired.it/internet/social-network/2017/03/31/social-network-in-italiafacebook-regna/ (08/10/2017 at 19:57)

[5] http://www.ansa.it/sito/notizie/tecnologia/software app/2017/06/01/instagram-ha14-milioni-utenti-in-italia_f761352c-b8c4-484e-ba65-58f82ae4dcf5.html $(08 / 10 / 2017$ at $19: 59)$ 